
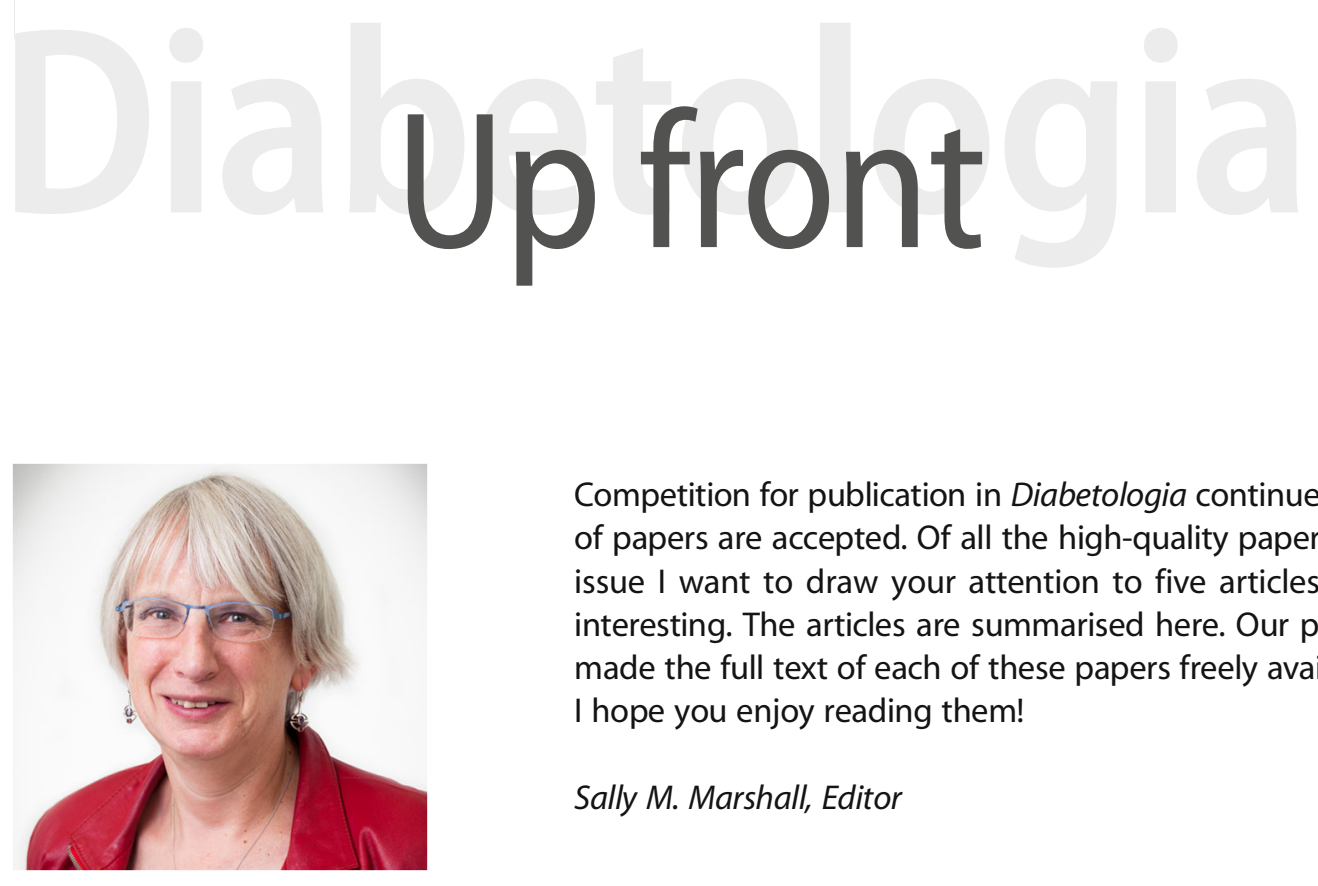

Competition for publication in Diabetologia continues to grow, and less than $20 \%$ of papers are accepted. Of all the high-quality papers that appear in this month's issue I want to draw your attention to five articles that I think are particularly interesting. The articles are summarised here. Our publisher, Springer, has kindly made the full text of each of these papers freely available.

I hope you enjoy reading them!

Sally M. Marshall, Editor

\section{Inter-organ communication and regulation of beta cell function}

Mehboob A. Hussain, Elina Akalestou, Woo-jin Song

Blood glucose levels are under constant tight regulation by the main pancreatic hormones glucagon and insulin, which are secreted by alpha and beta cells of the islets of Langerhans, respectively. While elevated blood glucose levels serve as the primary stimulus for insulin release, beta cell function is modulated by a multitude of signals that emanate from a variety of metabolically relevant tissues. In this issue, Hussain et al summarise current understanding of the interorgan communication and regulation of beta cell function. The review highlights recent advances in this field gained from genetic mouse models, which have allowed detailed interrogation of a variety of interorgan signalling mechanisms. The authors discuss recent tantalising advances on the differences between the main incretin hormones glucagon-like peptide 1 (GLP-1) and glucose-dependent insulinotropic polypeptide (GIP), as well as the interplay of a newly discovered tri-hormonal regulatory pathway between alpha and beta cells via liver-derived kisspeptin.
Accuracy of circulating adiponectin for predicting gestational diabetes: a systematic review and meta-analysis

Stamatina lliodromiti, Jennifer Sassarini, Thomas W. Kelsey, Robert S. Lindsay, Naveed Sattar, Scott M. Nelson

Universal screening for gestational diabetes (GDM) has not been implemented worldwide and targeted screening based on baseline risk factors identifies only around half of the women who will develop GDM. Biomarker-enhanced selection for screening is appealing, but fructosamine, $\mathrm{HbA}_{1 \mathrm{c}}$ and fasting glucose all perform relatively poorly. Circulating adiponectin is lower in women with GDM but it is unclear whether adiponectin can improve prediction of GDM prior to the development of overt disease. In this issue, lliodromiti et al present cumulative data suggesting that early pregnancy adiponectin, which can be measured in a non-fasting state, has a moderate predictive accuracy (sensitivity of $60.3 \%$ and specificity of $81.3 \%$ ) in identifying women who would develop GDM irrespective of other risk factors, and could thus facilitate targeted screening and early interventions for prevention of GDM. Evaluation of the role of adiponectin in composite models is warranted prior to its routine use in clinical practice. 
Associations of total amount and patterns of sedentary behaviour with type 2 diabetes and the metabolic syndrome: The Maastricht Study

Julianne D. van der Berg, Coen D. A. Stehouwer, Hans Bosma, Jeroen H. P. M. van der Velde, Paul J. B. Willems, Hans H. C. M. Savelberg, Miranda T. Schram, Simone J. S. Sep, Carla J. H. van der Kallen, Ronald M. A. Henry, Pieter C. Dagnelie, Nicolaas C. Schaper, Annemarie Koster

Studies have shown that physical activity plays a role in the development and management of type 2 diabetes mellitus. In addition to physical activity, there has been growing interest in the role of sedentary behaviour. In this issue, van der Berg et al examine cross-sectional associations between objectively assessed levels of sedentary behaviour and diabetes status. In almost 2,500 participants, the authors show that those with type 2 diabetes had 26 minutes more sedentary time per day than those without diabetes. Each additional hour of sedentary time was associated with increased odds of $22 \%$ for type 2 diabetes, independent of high-intensity physical activity. Future studies in participants with type 2 diabetes should be conducted to confirm these results. Nevertheless, these findings could have important implications for public health, as they suggest that sedentary behaviour may play a significant role in the development and prevention of type 2 diabetes.

\section{Association between sleeping difficulty and type 2 diabetes in women}

Yanping Li, Xiang Gao, John W. Winkelman, Elizabeth M. Cespedes, Chandra L. Jackson, Arthur S. Walters, Eva Schernhammer, Susan Redline, Frank B. Hu

Difficulty falling asleep or staying asleep, which affects $10-20 \%$ of the US population, has been reported in prior studies to increase risk of type 2 diabetes. However, whether the observed association is mediated by obesity, hypertension or depression, and whether it is independent of other sleep disorders, is unclear. In this issue, Li et al report data from a study led by Harvard University researchers following 133,353 women for up to 10 years. They observed a $45 \%$ higher risk of type 2 diabetes among women with sleeping difficulty. Around half (46\%) of the observed association was mediated by the effect of sleeping difficulty on risk of obesity, hypertension and depression. The combination of sleeping difficulty and other sleep conditions, including frequent snoring, sleep duration of $\leq 6$ hours, sleep apnoea and shift work, was associated with a fourfold increased risk of diabetes compared with those free from these conditions. The authors suggest that it is not just quantity of sleep, but also quality, that is important for reducing risk of type 2 diabetes.

\section{Stress resilience and subsequent risk of type 2 diabetes in $\mathbf{1 . 5}$ million young men}

Casey Crump, Jan Sundquist, Marilyn A. Winkleby, Kristina Sundquist

Psychosocial stress in adulthood has been associated with higher risk of type 2 diabetes, possibly mediated by both behavioural and physiological factors. However, it is unknown whether low stress resilience earlier in life is related to subsequent development of type 2 diabetes. In this issue, Crump et al report that low stress resilience at age 18 , based on standardised psychological assessments in 1.5 million male military conscripts in Sweden, is associated with a higher risk of developing type 2 diabetes in later life. The authors suggest that this may be partly because of unhealthy lifestyle behaviours that are more common with psychosocial stress, as well as stress-related hormonal and immunological factors. These findings suggest that low stress resilience may play an important long-term role in aetiological pathways for type 2 diabetes. Further elucidation of the underlying pathways may help improve preventive strategies by addressing important psychosocial factors.

All text supplied by the authors. 